\title{
The ultrasound diagnostic criteria for diastasis recti and its correlation with pelvic floor dysfunction in early postpartum women
}

\author{
Enze Qu^, Jiawei Wu, Man Zhang, Lili Wu, Ting Zhang, Jing Xu, Xinling Zhang \\ Department of Ultrasound, Third Affiliated Hospital of Sun Yat-sen University, Guangzhou, China \\ Correspondence to: Prof. Xinling Zhang, MD. Department of Ultrasound, Third Affiliated Hospital of Sun Yat-sen University, 600 Tianhe Road, \\ Guangzhou 510630, China. Email: zhx140630@163.com.
}

\begin{abstract}
Background: There has been a long-standing controversy about diastasis recti diagnostic criteria and its relation to pelvic floor dysfunction (PFD). This study aimed to establish ultrasound diagnostic criteria for diastasis recti and investigate the correlation between it and PFD in early postpartum females.

Methods: The inter-rectus distance (IRD) was measured at 3 locations in 116 healthy nulliparous females and 108 postpartum females. At the same time, they remained relaxed and then maintained a head-lift posture. The measurement for the 90th percentile was used to define the normal IRD in the nulliparous group. Afterward, the 108 postpartum females underwent an ultrasonographic examination of pelvic floor function. The correlations of these values with the IRD were then examined.

Results: We established the following ultrasonographic diagnostic criteria for diastasis recti: an IRD of $>2 \mathrm{~mm}$ at $3 \mathrm{~cm}$ below the umbilicus, $>20 \mathrm{~mm}$ at the umbilicus, and $>14 \mathrm{~mm}$ at $3 \mathrm{~cm}$ above the umbilicus. The IRD was positively correlated with body mass index $(\mathrm{BMI})$ in the nulliparous group $(\mathrm{r}=0.286, \mathrm{P}<0.01)$ and with age in the postpartum group $(\mathrm{r}=0.230, \mathrm{P}<0.05)$. The IRD was not relative to either the PFD ultrasound results or the clinical symptoms.
\end{abstract}

Conclusions: We established a set of ultrasonographic diagnostic criteria for diastasis recti at 3 locations along the umbilicus. There is no clear correlation between diastasis recti and PFD in early postpartum females.

Keywords: Diastasis recti; pelvic floor dysfunction (PFD); ultrasonography; diagnostic criteria

Submitted Apr 22, 2020. Accepted for publication Oct 09, 2020.

doi: 10.21037/qims-20-596

View this article at: http://dx.doi.org/10.21037/qims-20-596

\section{Introduction}

Diastasis recti is are commonly defined as the separation between the 2 sides of the rectus abdominis muscle or simply laxity of the linea alba (1). The condition is usually caused by abdominal expansion and hormonal changes during pregnancy. The diastasis recti incidence is $27-100 \%$ in women in late pregnancy and $30-68 \%$ in postpartum women $(2,3)$.
The inter-rectus distance (IRD) can be measured via clinical palpation or calipers (4-6). These measurement methods are relatively convenient but may be inaccurate due to the thickness of subcutaneous fat and relaxation of the abdominal wall $(7,8)$.

Ultrasonography is regarded as the most accurate method for measuring the IRD (7), and its results are consistent with the data derived from palpation, calipers,

^ ORCID: 0000-0001-6628-6302. 
and intraoperative measurements (9-11). However, the differentiation between a normal IRD and pathological separation remains controversial (12). Because there are few studies on diagnostic criteria for diastasis recti, it is difficult to define "normal" and "abnormal" conditions. Diastasis recti may develop at different locations above, at, and below the umbilicus, so the single-location diagnostic criteria used in most recent research and the lack of agreement on the diagnostic criteria could lead to inaccurate incidence and high false-negative rates.

The abdominal muscle is essential for maintaining body posture, torso and pelvic cavity stability, and abdominal organ support (13-15). Diastasis recti can cause several health complications, such as lower back pain and trunk muscle dysfunction $(16,17)$. Current treatments require a much more individualized diastasis recti map, including physiotherapy, prolotherapy, and surgical intervention.

Additionally, diastasis recti may have an impact on pelvic stabilization. In $2007,>60 \%$ of patients with diastasis recti were found to exhibit concurrent pelvic floor dysfunction (PFD) (18). In contrast, a prospective study published in 2017 revealed no association between diastasis recti and PFD (19). However, neither of these studies applied ultrasound to measure IRD, which may have led to a less precise result.

To define diastasis recti accurately, we first tried to set up the ultrasound diagnostic criteria. The second goal was to investigate the correlation between diastasis recti and PFD in early postpartum females.

\section{Methods}

\section{Study participants}

Between September 2017 and September 2018, 108 females with weak pelvic floor muscles determined by vaginal palpation, who underwent a routine postpartum examination of pelvic floor function were recruited to form the postpartum group, and 116 healthy nulliparous female volunteers from gynecological outpatient care were recruited to form the nulliparous group. The postpartum group periods were 3-12 months, which avoided the influence of natural recovery of diastasis recti (20). The ethics approval number for this trial is KY2016-203. All participants provided written informed consent. The exclusion criteria were as follows: (I) the inability to perform the head-lift posture or Valsalva maneuver; (II) poor healing of the cesarean section incision or local skin infection; (III) participation in abdominal muscle training or rehabilitation of the pelvic floor muscles after delivery; and (IV) avulsion of the levator ani causing severe pelvic organ prolapse (POP).

\section{Equipment and personnel}

The IRD was measured using the ProSound F75 system (with a 5-12 MHz linear probe) from Hitachi-Aloka (Tokyo, Japan) and the Supersonic Imagine system (with a 4-15 MHz linear probe) from Supersonic Imagine (Aix en Province, France). Ultrasonographic examinations of pelvic floor function were performed using the Voluson E8 system (with an RAB 4-8 MHz volume probe) from GE Medical Systems (Tiefenbach, Austria). A senior clinician with $>5$ years of experience in musculoskeletal ultrasonography performed the IRD measurements. Three senior clinicians with $>5$ years of experience in pelvic floor ultrasonography performed pelvic floor function examinations.

\section{Examination methods}

During the examination, each participant took a supine position on the bed with the head resting on a thin pillow and the legs fully extended. The abdomen was fully exposed from the xiphoid process to the pubic symphysis, and efforts were made to ensure participant warm. The IRD was measured at the following 3 locations, with the subject in either a resting position or the head-lift posture: $3 \mathrm{~cm}$ below the umbilicus, at the umbilicus, and $3 \mathrm{~cm}$ above the umbilicus. We used a ruler to locate the probe. The postpartum females were divided into different subgroups based on the results. For the head-lift posture, the subject's head was elevated from the pillow by approximately $10 \mathrm{~cm}$ while the shoulders remained on the bed. We measured the IRD 3 times at each location and then used the mean value. Additionally, we documented the deviation of the bilateral rectus abdominis from the linea alba and other notable features.

After urination, we asked the subject to perform pelvic muscle contractions with tomographic ultrasound imaging (TUI) to exclude levator avulsion. Afterward, the subject was asked to perform the optimal Valsalva maneuver (with a duration of $\geq 6$ seconds), during which the area of the levator hiatus and degree of organ prolapse in the anterior, central, and posterior compartments of the pelvic cavity were recorded (Figure 1). In the anterior compartment lies the bladder and the urethra; in the central compartment lies 

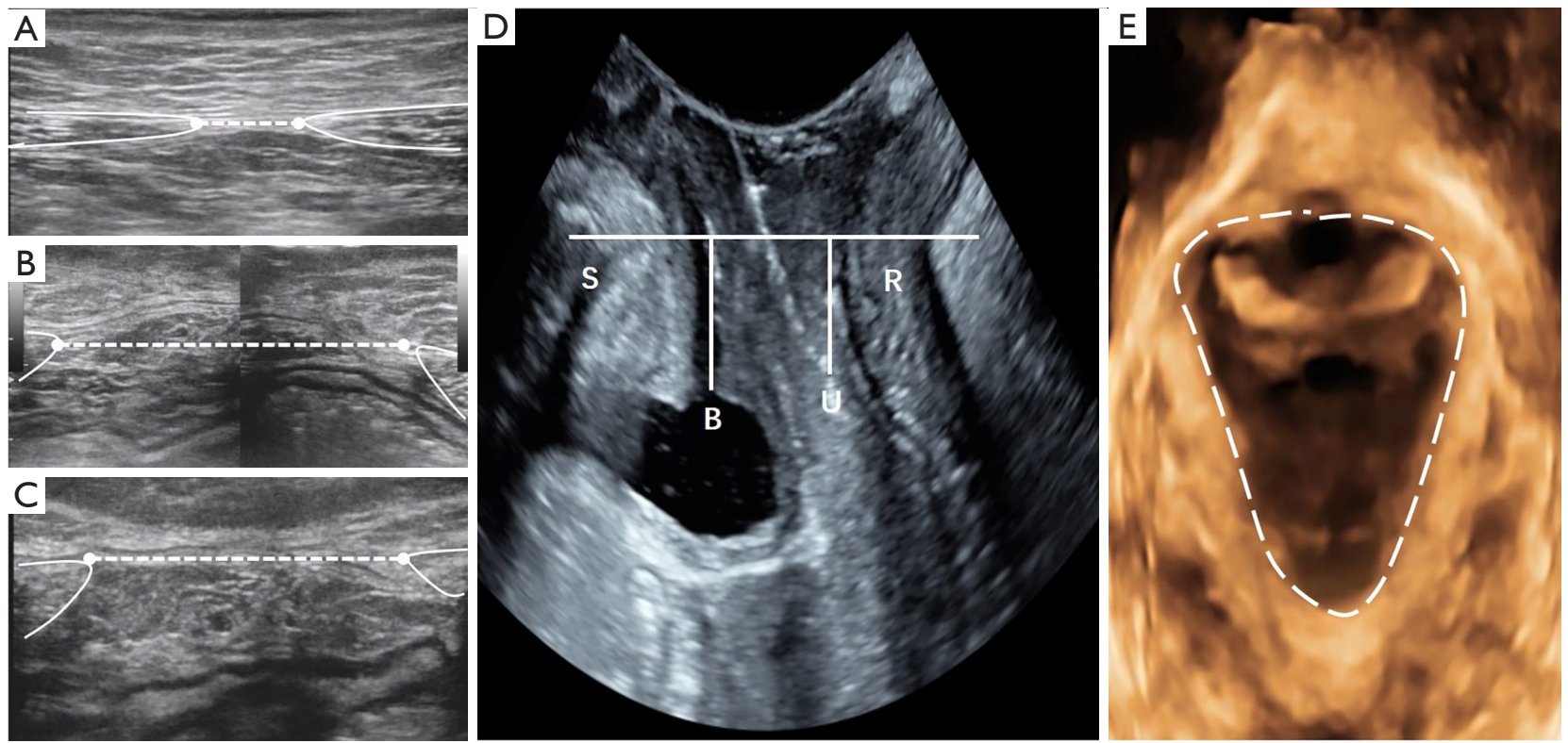

Figure 1 (A-C) Ultrasonographic images of the linea alba and the rectus muscles in a 20-year-old woman with a BMI of $20.5 \mathrm{~kg} / \mathrm{m} 2$, G1P1. (A) Transverse scan showing an IRD of $10 \mathrm{~mm}$ at $3 \mathrm{~cm} \mathrm{I-U;} \mathrm{(B)} 43 \mathrm{~mm}$ at the umbilicus; (C) $36 \mathrm{~mm}$ at $3 \mathrm{~cm} \mathrm{~S}-\mathrm{U}$; (D) the bladder neck was $12 \mathrm{~mm}$ above the line of reference, and the lowest point of the cervix was $10 \mathrm{~mm}$ above the line of reference. No significant degree of posterior compartment prolapse was observed; (E) the area of levator hiatus (dotted line) was $16 \mathrm{~cm} 2$. Note the distorted linea alba in (B,C). G1P1, first pregnancy; S, symphysis pubis; B, bladder; U, uterine; R, rectal ampulla, IRD, inter-rectus distance; I-U, infraumbilical; S-U, supraumbilical.

the uterus, cervix, and vagina; in the posterior compartment lies the rectum ampulla and anal canal. For prolapse quantification, we measured the distance from the bladder neck, the lowest point of the cervix, and the rectal ampulla to the reference line, which refers to the horizontal line through the inferior margin of the symphysis pubis (21). The hiatal area was measured in the rendered volume mode by tracing the levator muscles' inner margin. All 3 senior clinicians calculated the data offline independently using 4D View software (GE Healthcare, Tiefenbach, Austria).

During the measurements, we paid close attention to the following issues. First, the rectus sheath contains the anterior and posterior laminae. The 2 layers fuse in the abdominal midline to form the linea alba. The posterior lamina also creates the linea arcuata, located $4-5 \mathrm{~cm}$ below the umbilicus. Below the linea arcuata, the posterior lamina is absent. Therefore, we measured the width of the anterior lamina in all females. Second, if the medial edge of the rectus abdominis was difficult to identify, we asked the participant to perform the head-lift position, as it is easier to identify the boundary during muscle contractions. The female was then asked to lie flat before the measurement.
Third, we placed an adequate amount of gel on the umbilicus to avoid gas interference. Fourth, if the IRD was too wide to be displayed in a single image, the image assembly or wide-view imaging mode was used. Moreover, the IRD was sensitive to changes due to breathing in some females with severe diastasis recti. In these cases, we asked each female to hold her breath before the measurement.

\section{Statistical analysis}

The statistical software SPSS version 26.0 (SPSS Inc., Chicago, IL, USA) was used for the data analysis. The results are expressed as the mean \pm standard deviation, with 95\% confidence intervals (CIs). Differences between the 2 groups were compared using Student's $t$-test. A P value $<0.05$ was considered statistically significant, and $\mathrm{P}<0.01$ was considered highly significant. The value corresponding to the 90th percentile in the nulliparous group was adopted as the threshold of a "normal" IRD range (22). The interobserver reliability was determined by calculating intraclass correlation coefficients (ICCs) with 95\% CIs. Pearson's correlation coefficient or Spearman's correlation 
Table 1 General information about the nulliparous and postpartum groups

\begin{tabular}{lccc}
\hline Factors & Nulliparous & Postpartum & P value \\
\hline Age (years) & $29.51 \pm 4.56$ & $30.79 \pm 4.47$ & 0.378 \\
Height $(\mathrm{cm})$ & $159.82 \pm 5.20$ & $158.93 \pm 4.27$ & 0.053 \\
Weight $(\mathrm{kg})$ & $55.44 \pm 10.94$ & $57.20 \pm 6.28$ & 0.136 \\
BMl & $21.97 \pm 3.86$ & $22.63 \pm 2.29$ & 0.106 \\
\hline
\end{tabular}

Statistical method: Student's $t$-test. BMI, body mass index.

coefficient were used to assess the relationship between diastasis recti and age, body mass index (BMI), parity, the mean infant weight in the 2 groups, and the area of levator hiatus or the PFD results in the postpartum group. Because there are different morphologies of diastasis recti, the mean value does not exactly indicate abdominal muscle separation gravity. Therefore, we used the maximum value from the 3 locations at resting for the correlation analysis.

\section{Results}

A total of 116 women were included in the nulliparous group, and 102 women were included in the postpartum group (4 women were excluded due to poor Valsalva maneuver execution, and 2 women were excluded due to avulsion of the levator ani). Their general information is summarized in Table 1. No significant differences were identified between the groups in the general characteristics, including age, height, weight, and BMI. In the postpartum group, 49 participants' parity was 1, 46 women's parity was 2 , and 7 women's parity was 3 . The mean birth weight of the newborns was $3.18 \pm 0.46 \mathrm{~kg}$. Sixty-seven women were at 3 months postpartum, 26 were at 6 months postpartum, and 9 were at 12 months postpartum. The majority (59\%) of participants had a vaginal delivery, while $41 \%$ had a cesarean delivery. The average gestational weeks at delivery was $38.20 \pm 2.97$ weeks. According to the PFD questionnaire for the postpartum group, the clinical symptoms were also documented, and 49 women had no clinical symptoms. In comparison, 53 women presented various clinical symptoms, including mild stress incontinence $(\mathrm{n}=27)$, moderate stress incontinence $(\mathrm{n}=5)$, urge incontinence $(\mathrm{n}=3)$, dyspareunia $(n=2)$, constipation $(n=13)$, and frequent constipation $(n=10)$. The severity of stress incontinence was classified using the Ingelman-Sundberg classification of stress incontinence.

The IRD measurement results in the nulliparous group and the postpartum group are presented in Table 2 . Accordingly, the diagnostic criteria of diastasis recti were established as follows: (I) an IRD of $>2 \mathrm{~mm}$ at $3 \mathrm{~cm}$ below the umbilicus; (II) $>20 \mathrm{~mm}$ at the umbilicus; and (III) $>14 \mathrm{~mm}$ at $3 \mathrm{~cm}$ above the umbilicus. Based on these criteria, we divided the postpartum females into different groups: those who met only criterion (I) were classified as the subumbilical separation type; those who met only criterion (II) were classified as the umbilical separation type; those who met only criteria (III) were classified as the supraumbilical separation type; those who met criteria (I) + (II) were classified as umbilical \& subumbilical separation type; those who met criteria (II) + (III) were classified as umbilical \& supraumbilical separation type, and those who met the criteria at all 3 locations were classified as the complete separation type.

In contrast to the nulliparous group, the postpartum group's IRD values at all 3 locations were significantly different between the resting posture and the head-lift posture (Table 2). There were 18 females with an increased IRD in the head-lift position. A total of 9 participants did not meet any diagnostic criteria, yielding a diastasis recti incidence of $91.2 \%$ (93/102). Based on the rectus abdominis deviation, the participants were considered to have no deviation $(\mathrm{n}=57,61.3 \%)$, right deviation $(\mathrm{n}=6$, $6.4 \%)$, or left deviation $(n=30,32.3 \%)$. Based on the location of separation, the subjects were considered to have subumbilical separation $(\mathrm{n}=1,1.0 \%)$, umbilical separation $(\mathrm{n}=3,3.2 \%)$, supraumbilical separation $(\mathrm{n}=18,19.4 \%)$, umbilical \& subumbilical separation $(\mathrm{n}=2,2.2 \%)$, umbilical \& supraumbilical separation $(\mathrm{n}=15,16.1 \%)$, or complete separation $(\mathrm{n}=54,58.1 \%)$. A hernia of the linea alba had developed in 2 participants, yielding an incidence of $2.0 \%$ of this condition (23).

The correlations between the IRD and the participants' general information were as follows. The IRD was positively correlated with $\mathrm{BMI}(\mathrm{r}=0.286, \mathrm{P}<0.01)$ in the nulliparous group. The IRD was positively correlated with age $(\mathrm{r}=0.230, \mathrm{P}<0.05)$ but not with the mean infant weight, $\mathrm{BMI}$, or parity in the postpartum group. 
Table 2 IRD values for the nulliparous group and the postpartum group

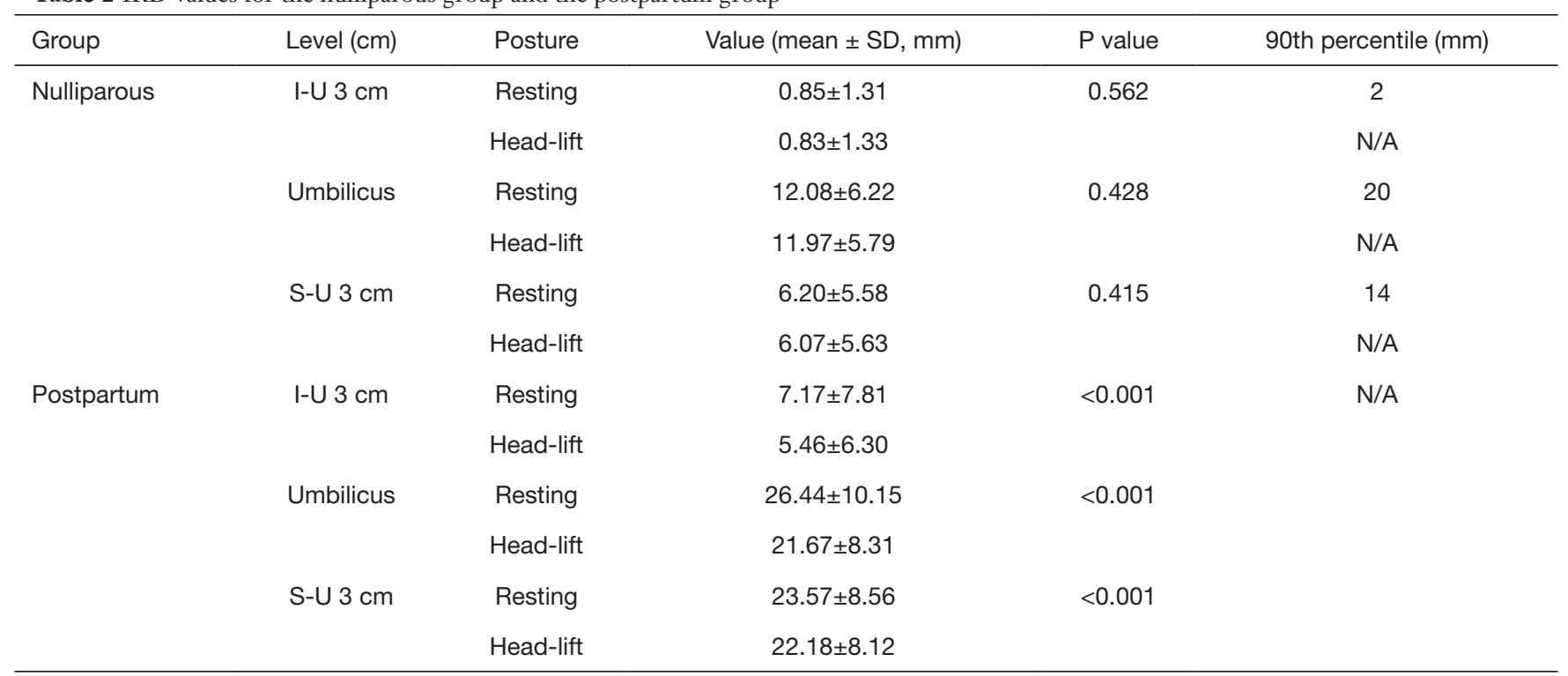

Statistical method: Student's $t$-test was used to compare the IRD values in the postpartum group at all 3 locations between the resting posture and the head-lift posture. S-U 3, $3 \mathrm{~cm}$ supraumbilical; I-U 3, $3 \mathrm{~cm}$ infraumbilical; IRD: inter-rectus distance.

Table 3 The ultrasound PFD results and IRD values for the postpartum group

\begin{tabular}{lccc}
\hline No. & Pelvic floor function & IRD resting (mean $\pm \mathrm{SD}, \mathrm{mm})$ & IRD head-lift $(\mathrm{mean} \pm \mathrm{SD}, \mathrm{mm})$ \\
\hline 19 & Normal & $28.1 \pm 10.5$ & $24.2 \pm 8.2$ \\
30 & 1 compartment involved $^{\star}$ & $27.2 \pm 9.5$ & $23.6 \pm 9.6$ \\
23 & 2 compartments involved & \\
30 & 3 compartments involved & $28.0 \pm 10.6$ & $23.8 \pm 9.7$ \\
\hline
\end{tabular}

*: an abnormal ultrasound result was found in only one compartment, e.g., bladder neck $<10 \mathrm{~mm}$ below the line of reference or the lowest point of the cervix $\leq 15 \mathrm{~mm}$ above the line of reference or rectal ampulla $\geq 15 \mathrm{~mm}$ below the line of reference. ${ }^{* *}$ : an abnormal ultrasound result was found in 2 compartments. ${ }^{* \star *}$ : an abnormal ultrasound result was found in 3 compartments. Statistical method: Spearman's correlation coefficient. IRD, inter-rectus distance; PFD, pelvic floor dysfunction.

The interobserver reliability of the ultrasound measurements across the 3 clinicians were 0.965 (95\% CI: 0.953-0.973), 0.987 (95\% CI: 0.964-0.993), and 0.949 (95\% CI: 0.815-0.978). Based on the ultrasound examination, 19 women were considered to have normal pelvic floor function, and 83 had PFD (1 compartment was involved in 30 cases, 2 compartments were involved in 23 cases, and 3 compartments were involved in 30 cases). The ultrasound PFD results and IRD values in the postpartum group are presented in Table 3. There were no significant differences between each group. The mean hiatal area of the levator ani was $24.67 \pm 6.97 \mathrm{~cm}^{2}$. However, no correlation was noted between the IRD and area of the levator hiatus. Moreover, the IRD values were not significantly different between participants with clinical symptoms and those without symptoms (Table 4).

\section{Discussion}

Although diastasis recti is a common condition in postpartum women, there are considerable discrepancies in the incidence, diagnostic criteria, and normal IRD range associated with different diagnostic approaches and cut-off values (24). In this study, the IRD was measured using high-frequency ultrasound, which has been demonstrated to be the most accurate and repeatable method. Our results revealed that IRD was positively correlated with BMI in healthy nulliparous women. This finding might explain the different 
Table 4 The relationship between the IRD and the clinical PFD symptoms

\begin{tabular}{|c|c|c|c|}
\hline $\mathrm{IRD}($ mean $\pm \mathrm{SD}, \mathrm{mm})$ & \multicolumn{2}{|c|}{ Pelvic floor dysfunction symptoms } & $P$ value \\
\hline Resting & $28.9 \pm 9.80$ & $27.3 \pm 9.24$ & 0.478 \\
\hline Head-lift & $25.2 \pm 8.47$ & $23.2 \pm 8.16$ & 0.530 \\
\hline
\end{tabular}

Statistical method: Student's $t$-test. IRD, inter-rectus distance; PFD, pelvic floor dysfunction; SD, standard deviation.

"normal" ranges reported by other research groups $(22,25)$.

Moreover, we believed that diastasis recti cannot be defined using a single-location diagnostic criterion because it may develop at different locations above, at, and below the umbilicus. Physiotherapists necessarily need to know not only whether a patient has diastasis recti, but also each patient's type precisely, because different varieties may require specific abdominal exercises or physiotherapy. Establishing such a set of criteria can help facilitate disease diagnosis and treatment.

There were no universally acceptable risk factors for diastasis recti; however, the putative etiological factors include an older delivery age, high BMI, multiple pregnancies, and macrosomia history $(2,24,26)$. Our results indicated that the severity of diastasis recti correlated with age and not with the mean birth weight of the newborn, $\mathrm{BMI}$, or parity.

The IRD values were smaller in the head-lift posture than in the resting position at all 3 locations in the postpartum group $(\mathrm{P}<0.001)$. These results were consistent with the conclusion that palpation may yield an underestimation of the degree of diastasis recti (24). The IRD can be measured when a subject assumes resting and head-lift postures. This process enables the detection of changes in IRD, which may be conducive to assessing lateral abdominal wall muscle function and guiding therapy (27-29). Interestingly, we found 18 participants had a larger IRD in the head-lift posture than in the resting posture. We presumed that these women might have required surgery to restore strength due to weak abdominal-pelvic fascia (27).

The group of PFD conditions includes defecation, urination, sexual activity disorders, POP, and pelvic cavity pain (30). Diastasis recti and PFD are both common conditions in pregnant and postnatal women. The abdominal wall muscle, pelvic fascia, and pelvic floor muscles collectively form the abdominal-pelvic cavity, which is an anatomical entity. Therefore, it is clinically imperative to examine the relationship between PFD and diastasis recti to develop clear guidelines for postpartum rehabilitation. Spitznagle et al. (18) published a retrospective study in 2007 in which only vaginal palpation was used to examine the strength of the pelvic floor muscles; they also did not use ultrasound to measure the IRD. In 2017, Bø et al. (19) conducted a prospective cohort study examining 300 postpartum women. Specifically, pelvic floor muscle strength was assessed using vaginal manometry, whereas the IRD was determined using the simple approach of palpation.

Pelvic floor ultrasonography is a safe and convenient method that can be used to evaluate anatomical and functional changes in the pelvic floor dynamically. It has been highly recommended to assess urinary dysfunction, anal incontinence, POP, protruding vaginal mass, and chronic pelvic pressure/discomfort (30). This study used ultrasound to assess the IRD and PFD, possibly generating more accurate and objective results than those in the studies discussed above. Our results revealed that there was no clear correlation between diastasis recti and PFD in early postpartum females. Additionally, there were no significant differences in the IRD values between participants with clinical symptoms and asymptomatic ones.

This study has the following limitations. First, the small sample sizes in both the nulliparous and postpartum groups might have led to inadequate accuracy of the diagnostic criteria. Second, we did not monitor weight gain or abdominal circumference changes during pregnancy in the postpartum group. Furthermore, the number of subjects across the different subgroups with different ultrasound PFD results was not well-matched, leading to inaccuracy of the results. In the future, we would like to include a larger sample size and use a univariate and multivariate linear regression model to investigate the association between independent variables and the IRD.

\section{Conclusions}

We established a set of ultrasonographic diagnostic criteria for diastasis recti at 3 locations along the umbilicus 
to facilitate disease diagnosis and develop appropriate physiotherapy treatment plans. The IRD was positively correlated with BMI in the nulliparous group and with age in the postpartum group. There is no clear correlation between diastasis recti and PFD in early postpartum females.

\section{Acknowledgments}

We would like to thank all participants who willingly consented to this study. Special thanks to Dr. Cui from Peking University 3rd Hospital for advice on the experimental design.

Funding: None.

\section{Footnote}

Conflicts of Interest: All authors have completed the ICMJE uniform disclosure form (available at http://dx.doi. org/10.21037/qims-20-596). The authors have no conflicts of interest to declare.

Ethical Statement: The study was approved by the ethics committee of The 2nd Affiliated Hospital of the Harbin Medical University of KY2016-203 and written all participants provided informed consent.

Open Access Statement: This is an Open Access article distributed in accordance with the Creative Commons Attribution-NonCommercial-NoDerivs 4.0 International License (CC BY-NC-ND 4.0), which permits the noncommercial replication and distribution of the article with the strict proviso that no changes or edits are made and the original work is properly cited (including links to both the formal publication through the relevant DOI and the license). See: https://creativecommons.org/licenses/by-nc-nd/4.0/.

\section{References}

1. Hsia $M$, Jones $S$. Natural resolution of rectus abdominis diastasis. Two single case studies. Aust J Physiother 2000;46:301-7.

2. Rett M, Braga M, Bernardes N, Andrade S. Prevalence of diastasis of the rectus abdominis muscles immediately postpartum: Comparison between primiparae and multiparae. Rev Bras Fisioter 2009;13:275-80.

3. Turan V, Colluoglu C, Turkyilmaz E, Korucuoglu U. Prevalence of diastasis recti abdominis in the population of young multiparous adults in Turkey. Ginekol Pol 2011;82:817-21.

4. Keeler J, Albrecht M, Eberhardt L, Horn L, Donnelly C, Lowe D. Diastasis recti abdominis: a survey of women's health specialists for current physical therapy clinical practice for postpartum women. J Womens Health Phys Ther 2012;36:131-42.

5. Gluppe SL, Hilde G, Tennfjord MK, Engh ME, Bø K. Effect of a Postpartum Training Program on the Prevalence of Diastasis Recti Abdominis in Postpartum Primiparous Women: A Randomized Controlled Trial. Phys Ther 2018;98:260-8.

6. Chiarello CM, Falzone LA, McCaslin KE, Patel MN, Ulery KR. The effects of an exercise program on diastasis recti abdominis in pregnant women. J Womens Health Phys Therap 2005;29:11-6.

7. van de Water AT, Benjamin DR. Measurement methods to assess diastasis of the rectus abdominis muscle (DRAM): A systematic review of their measurement properties and meta-analytic reliability generalisation. Man Ther 2016;21:41-53.

8. Mendes Dde A, Nahas FX, Veiga DF, Mendes FV, Figueiras RG, Gomes HC, Ely PB, Novo NF, Ferreira LM. Ultrasonography for measuring rectus abdominis muscles diastasis. Acta Cir Bras 2007;22:182-6.

9. Barbosa S, de Sa RA, Coca Velarde LG. Diastasis of rectus abdominis in the immediate puerperium: Correlation between imaging diagnosis and clinical examination. Arch Gynecol Obstet 2013;288:299-303.

10. Mota P, Pascoal AG, Sancho F, Bo K. Test-retest and intrarater reliability of 2-dimensional ultrasound measurements of distance between rectus abdominis in women. J Orthop Sports Phys Ther 2012;42:940-6.

11. Keshwani N, McLean L. Ultrasound imaging in postpartum women with diastasis recti: Intrarater between-session reliability. J Orthop Sports Phys Ther 2015;45:713-8.

12. Akram J, Matzen SH. Rectus abdominis diastasis. J Plast Surg Hand Surg 2014;48:163-69.

13. Fernandes da Mota PG, Pascoal AG, Carita AI, Bo K. Prevalence and risk factors of diastasis recti abdominis from late pregnancy to 6 months postpartum, and relationship with lumbo-pelvic pain. Man Ther 2015;20:200-5.

14. Michalska A, Rokita W, Wolder D, Pogorzelska J, Kaczmarczyk K. Diastasis recti abdominis - a review of treatment methods. Ginekol Pol 2018;89:97-101.

15. Dalal K, Kaur A, Mitra M. Correlation between diastasis rectus abdominis and lumbopelvic pain and dysfunction. 
Indian J Physiother Occup Ther 2014;8:210.

16. Thabet AA, Alshehri MA. Efficacy of deep core stability exercise program in postpartum women with diastasis recti abdominis: a randomised controlled trial. J Musculoskelet Neuronal Interact 2019;19:62-8.

17. Hills NF, Graham RB, McLean L. Comparison of Trunk Muscle Function Between Women With and Without Diastasis Recti Abdominis at 1 Year Postpartum. Phys Ther 2018;98:891-901.

18. Spitznagle TM, Leong FC, Van Dillen LR. Prevalence of diastasis recti abdominis in a urogynecological patient population. Int Urogynecol J Pelvic Floor Dysfunct 2007;18:321-8.

19. Bø K, Hilde G, Tennfjord MK, Sperstad JB, Engh ME. Pelvic floor muscle function, pelvic floor dysfunction and diastasis recti abdominis: Prospective cohort study. Neurourol Urodyn 2017;36:716-21.

20. Coldron Y, Stokes MJ, Newham DJ, Cook K. Postpartum characteristics of rectus abdominis on ultrasound imaging. Man Ther 2008;13:112-21.

21. Dietz HP, Franzcog DDU, Steensma AB. Atlas of Pelvic Floor Ultrasound. London: Springer, 2008:41-7, 63-7.

22. Beer GM, Schuster A, Seifert B, Manestar M, MihicProbst D, Weber SA. The normal width of the linea alba in nulliparous women. Clin Anat 2009;22:706-11.

23. Li M, Zhang L, Xu XJ, Shi Z, Zhao XM. CT and MRI features of tumors and tumor-like lesions in the abdominal wall. Quant Imaging Med Surg 2019;9:1820-39.

24. Benjamin DR, van de Water AT, Peiris CL. Effects of

Cite this article as: $\mathrm{Qu} \mathrm{E}, \mathrm{Wu} \mathrm{J}$, Zhang $\mathrm{M}, \mathrm{Wu} \mathrm{L}$, Zhang T, $\mathrm{Xu}$ J, Zhang X. The ultrasound diagnostic criteria for diastasis recti and its correlation with pelvic floor dysfunction in early postpartum women. Quant Imaging Med Surg 2021;11(2):706713. doi: 10.21037/qims-20-596 exercise on diastasis of the rectus abdominis muscle in the antenatal and postnatal periods: A systematic review. Physiotherapy 2014;100:1-8.

25. Liaw LJ, Hsu MJ, Liao CF, Liu MF, Hsu AT. The relationships between inter-recti distance measured by ultrasound imaging and abdominal muscle function in postpartum women: A 6-month follow-up study. J Orthop Sports Phys Ther 2011;41:435-43.

26. Candido G, Lo T, Janssen P. Risk factors for diastasis of the recti abdominis. Journal-Association of Chartered Physiotherapists in Women's Health 2005;97:49.

27. Lee D, Hodges P. New perspectives from the integrated systems model for treating women with pelvic girdle pain, urinary incontinence, pelvic organ prolapse, and diastasis rectus abdominis. In: Lee D, Hodges P. editors. Presented at the Associated Charter of Physiotherapists in Women's Health Conference. England, Bristol: Springer, 2013.

28. Pascoal AG, Dionisio S, Cordeiro F, Mota P. Inter-rectus distance in postpartum women can be reduced by isometric contraction of the abdominal muscles: A preliminary casecontrol study. Physiotherapy 2014;100:344-8.

29. Acharry N, Kutty RK. Abdominal exercise with bracing, a therapeutic efficacy in reducing diastasis-recti among postpartal females. Int J Physiother Res 2015;3:999-1005.

30. Pannu HK, Javitt MC, Glanc P, Bhosale PR, Harisinghani MG, Khati NJ, Mitchell DG, Nyberg DA, Pandharipande PV, Shipp TD, Siegel CL, Simpson L, Wall DJ, WongYou-Cheong JJ. ACR appropriateness criteria pelvic floor dysfunction. J Am Coll Radiol 2015;12:134-42. 